\title{
Primeros avances en la caracterización de vulcanitas de afloramientos de Antofagasta de la Sierra (prov. de Catamarca, Argentina)
}

\author{
Alejandra M. Elías* y Michael D. Glascock** \\ *Alejandra M. Elías, CONICET, Instituto Nacional de Antropología y \\ Pensamiento Latinoamericano, Secretaría de Cultura de la Nación, \\ Argentina. E-mail: alejandra.elias2@gmail.com \\ **Michael D. Glascock, Archaeometry Laboratory, University of Missouri \\ Research Reactor, USA. E-mail: glascockm@missouri.edu
}

\begin{abstract}
Resumen
En distintos sectores de la microrregión de Antofagasta de la Sierra (Provincia de Catamarca, Puna Meridional Argentina) se registran afloramientos de vulcanitas (rocas volcánicas con proporciones de vidrio menores a 80\%, sensu Aschero et al. 2002/2004) macroscópicamente muy similares.

Con el objetivo de diferenciarlas y aportar, de esta forma, a su identificación en el registro arqueológico, se aplicaron diversos métodos y técnicas sobre muestras de estas rocas procedentes de los distintos afloramientos. En esta instancia, se exponen los resultados obtenidos a partir de caracterización geoquímica por medio de Fluorescencia de Rayos $X$ de Energía Dispersiva.

Las vulcanitas procedentes de los diferentes afloramientos tienden a presentar disímiles concentraciones de ciertos elementos. Estos resultados son prometedores y estimulan a continuar investigando la aplicación de métodos geoquímicos en la diferenciación y determinación de la procedencia de estas rocas volcánicas. Cabe destacar que en el Noroeste Argentino los métodos geoquímicos fueron exclusivamente aplicados sobre una roca volcánica específica, la obsidiana, y aún no habían sido utilizados en otras rocas volcánicas anisótropas, con superficies, texturas y estructuras más irregulares.
\end{abstract}

Palabras claves: vulcanita 4; vulcanita 8; caracterización geoquímica; EDXRF; procedencia.

First advances in the geochemical characteristics of volcanics of outcrops of Antofagasta de la Sierra (province of Catamarca, Argentina)

\begin{abstract}
In different sectors of the Antofagasta de la Sierra micro-region (Catamarca Province, Southern Argentine Pune) outcrops of macroscopically very similar vulcanites (volcanic rocks with ratios of glass below $80 \%$, sensu Aschero et al. 2002/2004) have been recorded.

In order to differentiate between these and contribute to their identification in the archaeological record, various methods and techniques were applied to samples of these rocks. In this instance, the initial results obtained from geochemical characterization, through Energy Dispersive X-ray Fluorescence, are presented.

The vulcanites from different outcrops tend to show different concentrations for some elements. The results are promising and encourage further investigation into the application of geochemical methods for the differentiation and determination of the origin of these volcanic rocks. Note that in the Argentine Northwest geochemical methods have been exclusively applied to a particularly volcanic rock, obsidian, and have not yet been used on less homogeneous volcanic rocks with more irregular surfaces.
\end{abstract}

Keywords: vulcanite 4; vulcanite 8; geochemical characterization; EDXRF; provenance.

Conocer la base regional de recursos líticos y minerales es fundamental para aproximarse a la procedencia de los restos líticos relevados en los sitios arqueológicos. Esto contribuye en la evaluación de los patrones de movilidad de los grupos humanos, el acceso de los mismos a diversos espacios, las actividades de intercambio mantenidas con otros y las decisiones asociadas a la tecnología (Andrefsky 1994; Bamforth 1986, 1990; Carr 1994; Nelson 1991).
En la microrregión de Antofagasta de la Sierra (Provincia de Catamarca, Puna Meridional Argentina) existe una gran disponibilidad de recursos líticos y minerales que pudieron haber sido explotados y utilizados por las sociedades pasadas. En esta oportunidad nos focalizaremos en dos localidades, separadas entre sí por aproximadamente $15 \mathrm{~km}$, con afloramientos de rocas volcánicas: fondo de cuenca del Río Punilla y Campo Cortaderas. En ambas, 
se han registrado diversos afloramientos con variedades de vulcanitas macroscópicamente muy similares. En contribuciones previas nos extendimos en los criterios de diferenciación obtenidos a partir de análisis petrográficos de cortes delgados sobre muestras de vulcanitas relevadas en estos afloramientos (Elías et al. 2011). En este trabajo se desarrollan los resultados alcanzados a partir de la caracterización geoquímica de las muestras mencionadas. Se han determinado variaciones en las composiciones geoquímicas de las vulcanitas procedentes de los afloramientos del fondo de cuenca del Río Punilla y los de Campo Cortaderas, lo que lleva a sugerir que el análisis geoquímico conforma un método con potencial al momento de identificar y distinguir estas variedades de rocas en los conjuntos arqueológicos.

\section{Problema y objetivos}

En la microrregión de Antofagasta de la Sierra fueron varias las investigaciones que aportaron al conocimiento de la distribución de los recursos líticos que habrían estado disponibles para ser utilizados por las sociedades prehispánicas (Aschero et al. 1991; Aschero et al. 2002/2004; Escola 2003; Yacobaccio et al. 2004; entre otros). Veinticinco fuentes potenciales y efectivas fueron reconocidas, junto a 17 variedades de rocas/minerales empleados por las poblaciones: vulcanitas 1, 2, 3, 4, 5, 6, 7 y 8, vidrios volcánicos 1 y 2, obsidianas 1 1, 2a, 2b y 3, cuarcita, cuarzo y sílice (incluyendo ópalo y calcedonia) (Aschero et al. 2002/2004; Elías et al. 2011).

En esta oportunidad nos centraremos en las variedades de vulcanitas 4 y 8 , registradas en numerosos sitios arqueológicos de la microrregión correspondientes a diversos momentos temporales (Aschero et al. 1991; Babot et al. 2006; Escola 2003; Escola et al. 2006; Pérez 2005; Somonte y Cohen 2006) ${ }^{2}$. Cabe recordar que seguimos a Aschero et al. 2002/2004 en considerar vulcanitas a aquellas rocas volcánicas presentes en la microrregión de Antofagasta de la Sierra con porcentajes menores a $80 \%$ de vidrio.

La vulcanita 4 corresponde a una roca masiva de color negro y textura afanítica. Por su parte, la vulcanita 8 también es una roca afanítica que se presenta en forma de lajas, con distintos tamaños y grosores, de color gris y con 'manchas' blancas que corresponden a concentraciones de microlitos de plagioclasas (Elías et al. 2011). Inicialmente, estas variedades fueron identificadas en afloramientos del fondo de cuenca del Río Punilla, la primera en Los Negros y la segunda en Afloramientos

\footnotetext{
1 Aschero et al. 2002/2004 denominan 'vidrios volcánicos' a las rocas volcánicas con más de $80 \%$ de vidrio y de las que no es posible establecer si son ácidas o básicas. La denominación 'obsidianas' la reservan para los vidrios volcánicos ácidos.

2 En algunos casos se menciona la vulcanita 4 como dacita 4 o basalto variedad A. Ambas son denominaciones utilizadas previo a la nueva nomenclatura propuesta en Aschero et al. (2002/2004).
}

de Vulcanita 8 (Aschero et al. 2002/2004) (Figura 1). Posteriormente, rocas muy afines macroscópicamente a estas variedades (por su color, textura y forma de presentación) fueron identificadas en afloramientos del área arqueológica de Campo Cortaderas (Olivera et al. 2003/2005) (Figura 1).

Desde entonces, nos dedicamos a la búsqueda de criterios que posibilitaran la diferenciación de las vulcanitas halladas en los distintos afloramientos para su identificación en el registro arqueológico.

En contribuciones previas, como mencionamos, expusimos criterios de diferenciación obtenidos a partir del análisis petrográfico de cortes delgados de muestras de vulcanitas relevadas en los afloramientos del fondo de cuenca del Punilla y Campo Cortaderas (Elías et al. 2011). En este trabajo en particular, nos extenderemos en los resultados alcanzados a partir de la caracterización geoquímica de las muestras mencionadas. Los objetivos que perseguimos son:

a) contribuir a la evaluación del potencial de los análisis geoquímicos en la caracterización de rocas volcánicas con

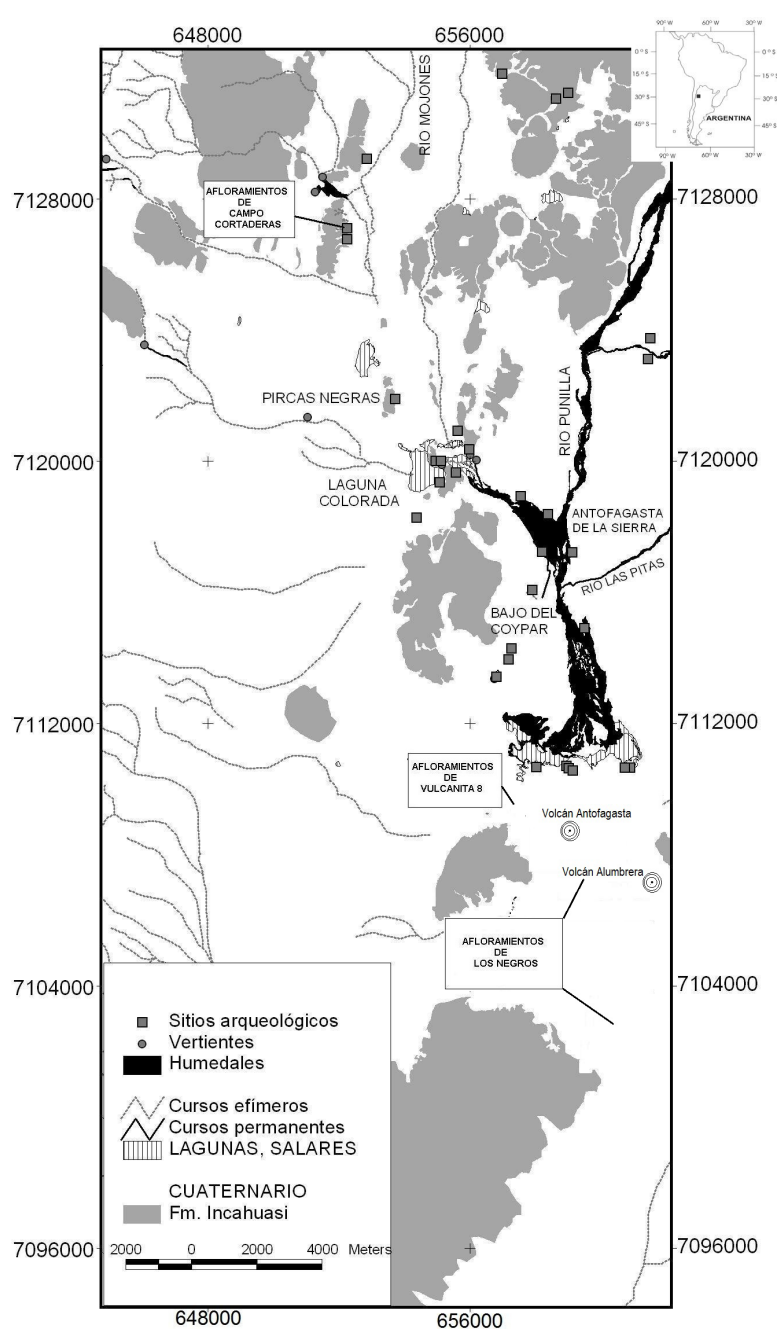

Figura 1. Ubicación de afloramientos de vulcanitas negras masivas y grises tabulares en Antofagasta de la Sierra (Elías et al. 2011).

Figure 1. Location of black massive and grey tabular vulcanite outcrops in Antofagasta de la Sierra (Elías et al. 2011). 
superficies y estructuras más irregulares que la obsidiana; b) continuar profundizando en el análisis comparativo de las vulcanitas 4 y 8 de los afloramientos del fondo de cuenca del Río Punilla y de Campo Cortaderas.

El objetivo último es seguir aportando a la comprensión y caracterización de la diversidad y complejidad de recursos líticos que habrían estado disponibles para las sociedades prehispánicas en la microrregión de Antofagasta de la Sierra.

\section{Breve descripción de los afloramientos}

En el fondo de cuenca del Punilla se identificaron concentraciones de vulcanita 4 en las márgenes de Los Negros. Estos conforman una multitud de coladas de rocas volcánicas ampliamente erosionadas y correspondientes a la Formación Incahuasi (Fase I), de edad Pleistocena Media (Aceñaloza et al. 1976). Se distinguieron dos sectores en los que la vulcanita 4 se presenta en forma de bloques y nódulos transportables y en los que se registran evidencias de las primeras etapas del proceso de reducción lítica (lascas primarias y secundarias, percutores de cuarcita). El sector Norte, ubicado aproximadamente a 6-8 km al Sur del poblado actual, y el sector Sur, ubicado 5,3 km hacia el sudeste del anterior (Escola 2000, 2003).

En lo que refiere a la vulcanita 8, afloramientos de esta variedad se emplazan a 5-7 km al Sur del poblado actual de Antofagasta de la Sierra, en la margen occidental de las coladas de los volcanes Antofagasta y La Alumbrera (Figura 1). Conforman una meseta volcánica asignada a la Formación Incahuasi, perteneciente a una fase de edad más reciente que los afloramientos de Los Negros (Fase 2 o 3) y donde no se han registrado indicios de actividades de talla y extracción en superficie (Escola 2003). Asimismo, hemos registrado afloramientos más acotados de vulcanita 8 en las márgenes de las coladas de Los Negros, al Sur de los anteriores (Elías et al. 2011).

Ahora bien, en el área arqueológica de Campo Cortaderas se identificaron cuatro sectores con afloramientos de vulcanita 4, distribuidos en sentido Norte-Sur e intercalados entre las abras en las cuales se disponen estructuras arqueológicas (Olivera et al. 2003/2005). Son afloramientos de edad Pleistocena media, asignados a la Formación Incahuasi (Fase I), y depósitos coluviales secundarios resultado de la meteorización y transporte de los primeros. La vulcanita 4 se presenta en forma de grandes bloques en los sectores más elevados y de nódulos de tamaños transportables hacia el piedemonte. Aunque aún no hemos realizado un registro sistemático de las evidencias arqueológicas en estos sectores, corresponde mencionar la presencia en algunos de ellos de lascas nodulares, núcleos y bloques con varios negativos de lascado (Elías et al. 2011).

Por su parte, la vulcanita 8 se encuentra en afloramientos al Norte y Sur de la localidad de Campo Cortaderas; los primeros conforman frentes de colada. Al igual que la vulcanita 8 del fondo de cuenca del Punilla, se presenta en forma de lajas, con tamaños y grosores diversos (Elías et al. 2011).

\section{Caracterización geoquímica y muestras}

Gran parte de los estudios geoquímicos sobre materiales líticos arqueológicos se han concentrado en la obsidiana (Glascock 2002; Shackley 1998). En comparación con esta roca, otros tipos de materiales líticos han sido pobremente estudiados aplicando los distintos métodos geoquímicos disponibles. En lo que respecta específicamente a otras rocas volcánicas (basaltos, andesitas, dacitas, riodacitas) son escasos los estudios realizados tanto en América como en el resto del mundo (Jones et al. 1997; Latham et al. 1992; Ogburn 2004; Seelenfreund et al. 2004, 2009; consultar también Haury [1994]:102). En el Noroeste Argentino se observa esta misma tendencia, habiéndose sólo recurrido a métodos geoquímicos para la caracterización de obsidianas (Chaparro 2007; Escola 2007; Escola et al. 1994; Flores y Morosi 2012; Scattolin y Lazzari 1997; Sprovieri y Glascock 2007; Tarragó et al. 1997; Yacobaccio et al. 2004; entre otros).

Ahora bien, en el ámbito de la geología estos métodos y técnicas han sido aplicados sobre diversas rocas volcánicas, no sólo obsidianas. Es así que, desde la década del 60, los geólogos han empleado la Fluorescencia de Rayos X de Energía Dispersiva (EDXRF) (Jones et al. 1997). A esta técnica recurrimos para la caracterización de las muestras de vulcanitas objeto de esta investigación.

Los análisis fueron realizados en el Missouri University Research Reactor (MURR). Se utilizó un espectrómetro ElvaX con tubo de tungsteno. El espectrómetro fue calibrado en base a información de muestras geológicas de obsidianas con concentraciones de elementos conocidas, obtenidas por Análisis de Activación Neutrónica (NAA) y Fluorescencia de Rayos X (XRF). Se consideraron 11 elementos (Tabla 1): potasio (K), calcio (Ca), titanio (Ti), hierro (Fe), cinc (Zn), galio (Ga), rubidio $(\mathrm{Rb})$, estroncio $(\mathrm{Sr})$, itrio $(\mathrm{Y})$, circonio $(\mathrm{Zr})$ y niobio $(\mathrm{Nb})$. Las concentraciones de estos fueron medidas en partes por millón (ppm) y determinadas a partir del software de análisis espectral ElvaX (Glascock et al. 1998).

Desarrollaremos brevemente las características generales de la técnica de XRF y las posibilidades y limitaciones que ofrece al aplicarla, con el objetivo de determinar diferencias geoquímicas y procedencia, sobre muestras no modificadas de rocas volcánicas con superficies y estructuras más irregulares que la obsidiana.

La espectrometría de XRF se basa en la detección de rayos $X$ de distintas energías emitidas por átomos que han sido excitados por rayos $X$ de alta energía (radiación X primaria 
o incidente). Frente a esta radiación los electrones de capas interiores del átomo son expulsados y los espacios dejados por estos ocupados por electrones de capas más externas. La energía es conservada en este proceso por la emisión de radiación de rayos $\mathrm{X}$ (radiación secundaria o fluorescencia) con niveles de energías característicos de los elementos presentes en la muestra (los niveles de energía son únicos para cada elemento). Al medir la intensidad de los rayos $X$ emitidos se puede determinar la cantidad/concentración de cada elemento en la muestra (Glascock et al. 1998; Herz y Garrison 1998; Jones et al. 1997; Pollard et al. 2007).

La XRF generalmente requiere que las muestras irradiadas presenten superficies relativamente lisas y uniformes y que estén compuestas por partículas pequeñas en comparación con la distancia que los rayos $X$ de interés pueden viajar antes de ser reabsorbidos por la muestra. Estas distancias van desde micrones para elementos livianos como sodio y magnesio, a varios milímetros para un elemento pesado como el bario. El análisis cuantitativo, es decir determinar la cantidad/concentración de cada elemento, usualmente no es posible para materiales que no cumplen con estos requisitos. En estos casos, se requiere que la muestra sea molida a un polvo de tamaño de micrones y reducida a vidrio. Esto constituye un método destructivo no susceptible de ser elegido por los arqueólogos (Latham et al. 1992).

Ahora bien, Latham et al. (1992) sugieren que información útil y confiable puede ser obtenida, en determinadas situaciones, a partir de muestras enteras. Uno de estos casos son los elementos con alto número atómico en una matriz de elementos livianos, por ejemplo $\mathrm{Zr}$ y $\mathrm{Sr}$ en una roca compuesta fundamentalmente de oxígeno (O), silíce (Si) y aluminio (Al). Pese a que problemas geométricos asociados a la irregularidad de las muestras pueden introducir errores sistemáticos en la medición de elementos pesados con números atómicos similares, por ejemplo Zr y Sr, los errores son esencialmente los mismos y los autores plantean que pueden ser suprimidos recurriendo al índice de las intensidades de rayos $X$ de los dos elementos.

Jones et al. (1997) señalan, en base a sus investigaciones geoquímicas aplicando XRF sobre andesitas y dacitas, que realizar estimaciones precisas de las concentraciones elementales requiere aplicar al menos una técnica semidestructiva de preparación de las muestras (por ejemplo, pulir las superficies de las rocas). Sin embargo, también indican en su estudio que, en base a la composición geoquímica y las intensidades relativas del espectro de líneas de rayos $X$, las fuentes geológicas pudieron ser discriminadas en ejemplares sin modificar.

Estas consideraciones nos llevan a justificar la aplicación de EDXRF en las muestras de vulcanitas, no modificadas y sin ningún tipo de tratamiento previo consideradas en esta instancia. Contamos con un total de 16 muestras recogidas en distintos sectores de los afloramientos, seleccionadas con un diseño de muestreo al azar. Cabe aclarar que fueron tomadas de los nódulos y lascas de los que se obtuvieron cortes delgados para estudios petrográficos (Elías et al. 2011). Diez muestras corresponden a vulcanitas negras masivas y las seis restantes a vulcanitas grises tabulares (Tabla 1).

Entre las primeras, cuatro muestras provienen de los afloramientos de la Formación Incahuasi (Fase I) del área de Campo Cortaderas: dos de la fuente 1 (AEB003 y AEB004) y las restantes de las fuentes 2 (AEB002) y 3 (AEB001) (Tabla 1).

Las seis muestras restantes de vulcanita negra masiva provienen de los afloramientos del fondo de cuenca del Río Punilla: cinco fueron relevadas en distintos puntos de las márgenes de Los Negros (AEB005, AEB006, AEB007, AEB008, AEB009) y la restante (AEB011) en los Afloramientos de Vulcanita 8 (Tabla 1).

En lo que respecta a las vulcanitas grises tabulares, tres de las seis muestras consideradas proceden de afloramientos del fondo de cuenca del Punilla: dos de los Afloramientos de Vulcanita 8 ubicados al Norte (AEB012 y AEB013) y una (AEB015) de la manifestación más restringida espacialmente de esta variedad en las márgenes de Los Negros (Tabla 1).

Otras tres muestras de vulcanitas grises tabulares (AEB016, AEB018 y AEB020) fueron relevadas en los afloramientos de Campo Cortaderas, en distintos puntos de los frentes de coladas ubicados al Norte del área (Tabla 1).

Somos conscientes de que las muestras con las que contamos no son ampliamente representativas de las poblaciones a comparar. En general, los afloramientos resultantes de actividades volcánicas están compuestos por distintas coladas, entre y dentro de las cuales las rocas pueden presentar variación (macroscópica, petrográfica, geoquímica), dependiendo de los distintos tiempos de enfriamientos de las lavas y el ambiente circundante. Por ello, es necesario contar con un número significativo de muestras procedentes de distintas coladas y sectores de los afloramientos para disponer del rango de variabilidad en los mismos y lograr una caracterización promediada (Glascock et al. 1998; Haury 1994; Jones et al. 1997; Latham et al. 1992; Shackley 1998). En este sentido, el estudio realizado por nosotros es un proyecto piloto y los resultados alcanzados son iniciales, por lo que deberán ser corroborados a futuro recurriendo a un número más amplio de muestras. Sin embargo, cabe adelantar que las tendencias derivadas de ellos son muy prometedoras.

Finalmente, en el manejo estadístico de los datos se recurrió a gráficos de dispersión y bivariados de elementos, así como a estadística multivariada, más específicamente Análisis de Componentes Principales (RQ-mode). En esta última instancia sólo se consideraron los siguientes elementos: Fe, Zn, Rb, Sr, Y, Zr y Nb. 


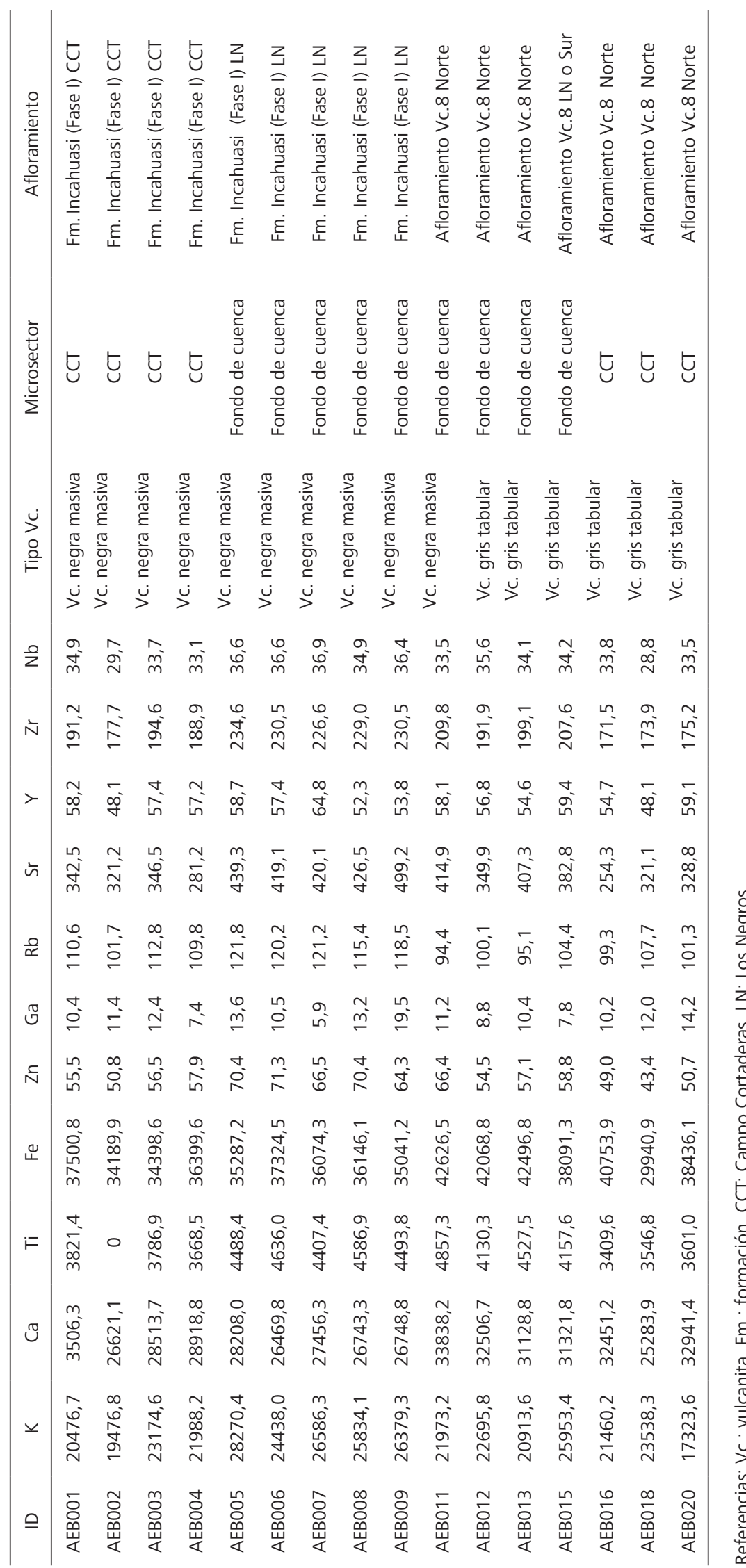

Tabla 1. Procedencias y concentraciones elementales (ppm) de muestras de vulcanitas negras masivas y grises tabulares.

Table 1. Sources and elemental concentrations of black massive and grey tabular vulcanite samples.

\section{Resultados}

Las muestras de vulcanitas negras masivas procedentes de Los Negros y Campo Cortaderas se segregan en función de las concentraciones de $\mathrm{Zr}$, $\mathrm{Sr}, \mathrm{Zn}$ y $\mathrm{Rb}$.

En los gráficos de dispersión bivariados se observa que las muestras se agrupan en función de sus afloramientos de origen. Por ejemplo, al considerar Zr vs. Zn y $\mathrm{Rb}$ vs. Sr vemos que las vulcanitas procedentes de Campo Cortaderas, si bien presentan variabilidad entre ellas, se separan claramente de las de Los Negros. El ejemplar procedente del Afloramiento de Vulcanita 8 se aísla de ambos conjuntos (Figuras 2).

Finalmente, se aplicó Análisis de Componentes Principales (RQmode) considerando los siguientes elementos: Fe, Zn, Rb, Sr, Y, Zr y Nb. Las vulcanitas negras masivas de Los Negros se agrupan claramente con altas cantidades de $\mathrm{Zn}, \mathrm{Zr}$, Rb y Sr. Las de Campo Cortaderas, aunque menos claramente agrupadas, se aíslan de las anteriores, con concentraciones menores de los elementos mencionados (Figura 3).

En lo que respecta a las vulcanitas grises tabulares, las muestras del fondo de cuenca del Punilla (de los Afloramientos de Vulcanita 8 al Oeste de los volcanes Antofagasta y La Alumbrera y el ubicado más al Sur) y de Campo Cortaderas se segregan en función de sus cantidades de Zr y Zn. Esto se observa, aunque las muestras no se agrupan claramente, en las Figuras 3 y 4 . Las vulcanitas grises tabulares de los afloramientos del fondo de cuenca del Punilla tienden a presentar mayores concentraciones de Zr y Zn.

\section{Conclusiones}

Geoquímicamente se alcanzaron resultados que aunque preliminares son prometedores. A pesar de que las diferencias geoquímicas entre las rocas procedentes de los distintos afloramientos considerados no son 

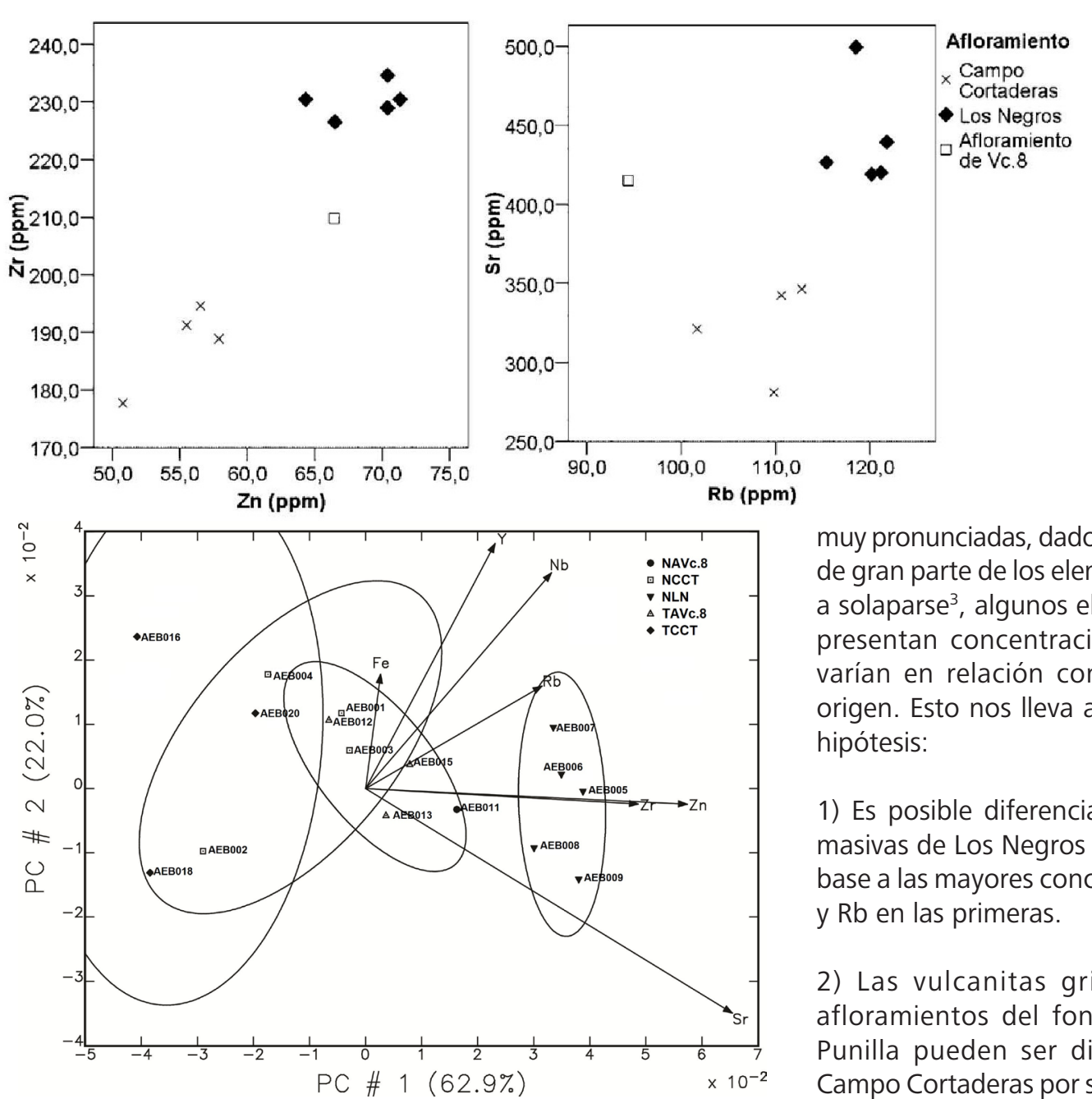

Figura 3. ACP de muestras de vulcanitas negras masivas y grises tabulares. Figure 3. ACP of black massive and grey tabular vulcanite samples. Referencias: NAVc.8: vulcanita negra masiva de Afloramientos de Vulcanita 8; NCCT: vulcanita negra masiva de Campo Cortaderas; NLN: vulcanita negra masiva de Los Negros; TAVc.8: vulcanita gris tabular de Afloramientos de Vulcanita 8; TCCT: vulcanita gris tabular de Campo Cortaderas.

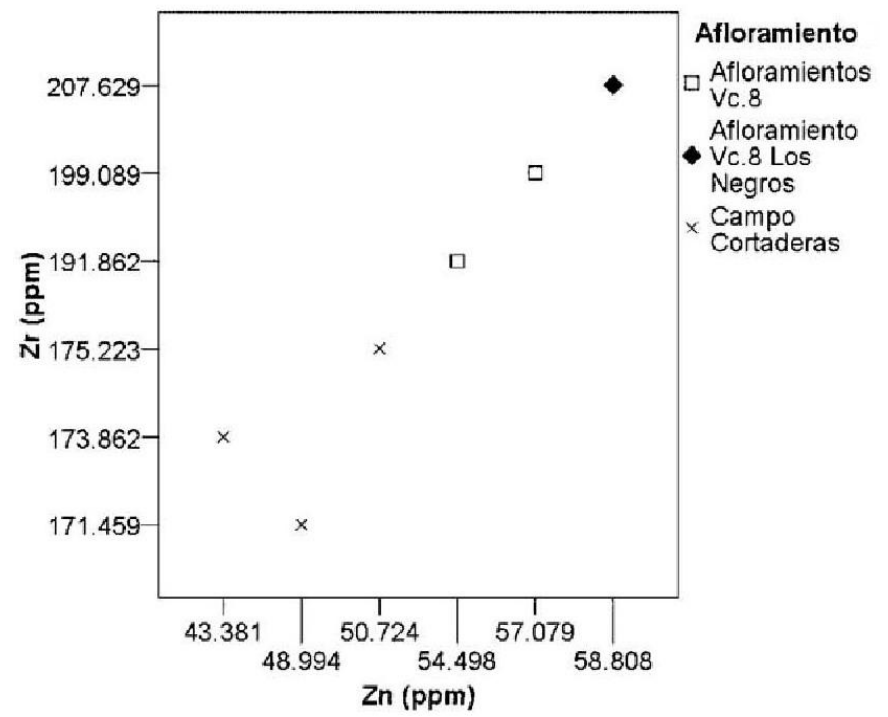

Figura 4. Gráficos bivariados de concentraciones de Zr vs. Zn en muestras de vulcanitas grises tabulares.

Figure 4. Bivariate plot of $\mathrm{Zr}$ vs. Zn in grey tabular vulcanite samples. hipótesis: y $\mathrm{Rb}$ en las primeras. elevadas de Zr y Zn. rocas de los afloramientos.
Figura 2. Gráficos bivariados de concentraciones de $\mathrm{Zr}$ vs. Zn y Sr vs. Rb en muestras de vulcanitas negras masivas. Figure 2. Bivariate plots of $\mathrm{Zr}$ vs. Zn and Sr vs. Rb in black massive vulcanite samples.

muy pronunciadas, dado que las concentraciones de gran parte de los elementos medidos tienden a solaparse ${ }^{3}$, algunos elementos ( $\left.\mathrm{Zr}, \mathrm{Zn}, \mathrm{Sr}, \mathrm{Rb}\right)$ presentan concentraciones diferenciales que varían en relación con los afloramientos de origen. Esto nos lleva a plantear las siguientes

1) Es posible diferenciar las vulcanitas negras masivas de Los Negros y Campo Cortaderas en base a las mayores concentraciones de $\mathrm{Zr}, \mathrm{Sr}, \mathrm{Zn}$

2) Las vulcanitas grises tabulares de los afloramientos del fondo de cuenca del Río Punilla pueden ser diferenciadas de las de Campo Cortaderas por sus concentraciones más

Estas hipótesis deberán ser corroboradas a futuro con la incorporación de un número mayor de muestras, sin dejar de advertir la necesidad de caracterizar también geoquímicamente artefactos arqueológicos y comparar los resultados con las tendencias obtenidas por medio de este método entre las muestras de

En el año 2001 se detectó que a aproximadamente $15 \mathrm{~km}$ de los afloramientos de vulcanitas 4 y 8 del fondo de cuenca del Río Punilla (Escola 2000) existían, en el área arqueológica de Campo Cortaderas, afloramientos de rocas muy similares (Olivera et al. 2003/2005). De esta forma, un nuevo lugar de procedencia de estas variedades se incorporó a los ya conocidos (Aschero et al.

\footnotetext{
${ }^{3}$ Esto podría relacionarse con el hecho de que los afloramientos de Campo Cortaderas y Los Negros y afloramientos de vulcanita 8 del fondo de cuenca del Punilla serían resultado de un mismo evento volcánico (Formación Incahuasi, Fase I) y compartirían un mismo magma de origen, aunque sólo estudios específicos respaldarán esta sugerencia (Pablo Tchilinguiran, comunicación personal 2010).
} 
2002/2004) y con esto se amplió el número de fuentes potenciales de las que procederían las vulcanitas $4 \mathrm{y}$ 8 identificadas en distintos sitios arqueológicos de la microrregión de Antofagasta de la Sierra.

Luego de descartar, en forma sistemática, que las diferencias macroscópicas entre muestras de rocas relevadas en los distintos afloramientos pudieran ser diagnósticas (Elías et al. 2011), nos concentramos en la búsqueda de otros criterios de discriminación. Inicialmente, recurrimos a la caracterización petrográfica de cortes delgados. Ésta aportó criterios de distinción entre las vulcanitas negras masivas y las vulcanitas grises tabulares de los distintos afloramientos. En lo que refiere a las primeras: a) las muestras de Los Negros tienden a presentar $\square 80 \%$ de vidrio en pasta respecto a las de Campo Cortaderas, b) variedades con $<70 \%$ de vidrio podrían provenir tanto de Campo Cortaderas como de los afloramientos del fondo de cuenca del Punilla, y c) ejemplares con $<30 \%$ de vidrio en pasta procederían de Campo Cortaderas. Por su parte, las muestras de vulcanitas grises tabulares son muy similares, exceptuando las dimensiones de los cristales de piroxenos (en pasta y fenocristales), los que presentan tamaños menores entre las muestras del fondo de cuenca del Punilla que entre las de Campo Cortaderas.

En este trabajo, nos centramos en aplicar un método que, hasta el momento, sólo fue empleado en el Noroeste Argentino en la determinación de procedencia de obsidianas. Los resultados alcanzados, acompañando a lo observado petrográficamente, indican diferencias geoquímicas entre las muestras de vulcanitas 4 y 8 de los distintos afloramientos de la microrregión. Concluimos destacando el potencial de profundizar en esta línea de investigación, lo que podría ampliar los métodos disponibles para acercarnos a la procedencia de rocas volcánicas no totalmente hialinas relevadas en el registro arqueológico del Noroeste Argentino.

Buenos Aires, Julio de 2012

\section{Agradecimientos}

A la comunidad de Antofagasta de la Sierra. Este trabajo fue realizado en el marco de los siguientes subsidios: "Ambiente y Recursos en Sociedades Arqueológicas de la Puna Argentina" (UBACyT, F122), "Cambio Social, Manejo de Recursos y Paleoambientes en la Puna de Atacama durante el Holoceno" (ANPCYT, PICT N² 26023) y "Cultura, Paleoambiente y Disponibilidad de Recursos en Sociedades Arqueológicas de la Puna Argentina" (PIP CONICET 6333). Finalmente, a los evaluadores cuyos comentarios y aportes conformaron una importante contribución.

\section{Bibliografía}

Aceñolaza, F. G., A. J. Toselli y O. E. González. 1976.
Geología de la región comprendida entre el salar del Hombre Muerto y Antofagasta de la Sierra, provincia de Catamarca. Revista de la Asociación Geológica Argentina 31(2):127-136.

Andresfky, W. Jr. 1994. Raw material availability and the organization of technology. American Antiquity 59:21-34.

Aschero, C. A., D. Elkin y E. Pintar. 1991. Aprovechamiento de recursos faunísticos y producción lítica en el precerámico tardío. Un caso de estudio: Quebrada Seca 3 (Puna Meridional Argentina). Actas XII Congreso Nacional de Arqueología Chilena, Tomo II, pp. 101-114, Santiago de Chile.

Aschero, C. A., P. S. Escola, S. Hocsman y J. G. Martínez. 2002/2004. Recursos líticos en la escala microrregional Antofagasta de la Sierra, 1983-2001. Arqueología 12:936.

Babot, M., C. A. Aschero, S. Hocsman, C. Haros, L. González Baroni y S. Urquiza. 2006. Ocupaciones agropastoriles en los sectores intermedios de Antofagasta de la Sierra (Catamarca): un análisis desde Punta de la Peña 9. Comechingonia 9:57-78.

Bamforth, D. 1986. Technological efficiency and tool curation. American Antiquity 51:38-50.

1990. Settlement, raw material and lithic procurement in the Central Mojave Desert. Journal of Anthropological Archaeology 9:70-104.

Carr, P. J. 1994. The organization of technology: impact and potencial. En P. J. Carr (ed.), The organization of north american prehistoric chipped stone tool technologies, Capítulo 1, pp. 1-8, International Monographs in Prehistory, Ann Arbor.

Chaparro, Ma. G. 2007. Preferencias en el manejo cotidiano de rocas. Los artefactos líticos de los asentamientos estatales del ur de Quebrada de Humahuaca y el valle Calchaquí medio (Argentina). V. Williams y B. Cremonte (eds.), Al borde del imperio. Paisajes sociales en áreas periféricas del Qollasuyu. En prensa.

Church, T. (ed.). 1994. Lithic resource studies: a sourcebook for archaeologists, Department of Anthropology, University of Tulsa, Oklahoma.

Elías, A. M., P. Tchillinguirian y P. S. Escola. 2011. De lo macroscópico a lo microscópico: vulcanitas similares de procedencias diversas (Antofagasta de la Sierra, Provincia de Catamarca, Puna Meridional Argentina). Intersecciones en Antropología 12:207-220.

Escola, P. S. 2003. Disponibilidad de recursos líticos y fuentes de aprovisionamiento en un sector de la Puna Meridional. Mundo de Antes 3:65-84. 
Escola, P. S. 2007. Obsidianas en contexto: tráfico de bienes, lazos sociales y algo más. En V. Williams, B. Ventura, A. Callegari y H. Yacobaccio (eds.), Sociedades precolombinas surandinas. Temporalidad, interacción y dinámica cultural del NOA en el ámbito de los Andes Centro-Sur, pp. 73-87, Buenos Aires.

Escola, P. S., C. Vázquez y F. Momo. 1994. Análisis de procedencia de artefactos de obsidiana: vías metodológicas de acercamiento al intercambio. Revista del Museo de Historia Natural de San Rafael 13(1):307311.

Escola, P. S., A. M. Elías y L. S. Paulides. 2006. Bajo del Coypar II: tendencias tecnológicas para el Tardío de Antofagasta de la Sierra (Catamarca). Werken 8:5-23.

Flores, M. C., M. Morosi. 2010. ¿De dónde vienen?: obsidianas de la localidad de Azampay (Depto. de Belén, Catamarca). En S. Bertolino, R. Cattáneo y A. Izeta (eds.), La arqueometría en Argentina y Latinoamérica, pp.177182, Editorial de la Facultad de Filosofía y Humanidades de la Universidad Nacional de Córdoba, Córdoba.

Glascock, M. D. 2002. Introduction: geochemical evidence for long-distance exchange. En M. D. Glascock (ed.), Geochemical evidence for long-distance exchange, Capítulo 1, pp. 1-12, Bergin and Garvey, Westport.

Glascock, M. D., G. E. Braswell y R. H. Cobean. 1998. A systematic approach to obsidian source characterization. En M. S. Shackley (ed.), Archaeological obsidian studies: method and theory, Capítulo 2, pp. 15-65, Plenum Press, New York.

Haury, C. E. 1994. Defining lithic procurement terminology. En T. Church (ed.), Lithic resource studies: a sourcebook for archaeologists, Capítulos 3-7, pp. 26-229, Department of Anthropology, University of Tulsa, Oklahoma.

Herz, N., E. G. Garrison. 1998. Geological methods for archaeology, Oxford University Press, Oxford.

Jones, G. T., D. G. Bailey y C. Beck. 1997. Source provenance of andesite artefacts using non-destructive XRF analysis. Journal of Archaeological Science 24:929943.

Latham, T. S., P. A. Sutton y K. L. Verosub. 1992. Nondestructive XRF characterization of basaltic artifacts from Truckee, California. Geoarchaeology 7(2):81-101.

Nelson, M. C. 1991. The study of technological organization. Archaeological Method and Theory Vol. 3: 57-100.

Ogburn, D. E. 2004. Evidence for long-distance transportation of building stones in the Inka Empire, from Cuzco, Perú to Saraguro, Ecuador. Latin American Antiquity 15:419-439.

Olivera, D. E., S. Vigliani, A. M. Elías., L. G. Grana y P. Tchilinguirian. 2003/2005. La ocupación Tardío-Inka en la Puna Meridional: el sitio Campo Cortaderas. Cuadernos del Instituto Nacional de Antropología 20:257-277.

Pérez, S. 2005. Análisis de microdesgaste por uso en palas y/o azadas líticas de Antofagasta de la Sierra (Pcia. De Catamarca). Aportes para su interpretación funcional. Hombre y Desierto 12:23-46.

Pollard, M., C. Batt, B. Stern y S. M. M. Young (editores). 2007. Analytical chemistry in archaeology, Cambridge University Press, Cambridge.

Scattolin, Ma. C., M. Lazzari. 1997. Tramando redes: obsidianas al oeste del Aconquija. Estudios Atacameños $14: 211-222$.

Seelenfreund, A., C. Sinclaire, P. de Souza, Ma. I. Dinator, E. Fonseca, M. Chesta y J. Morales. 2004. Caracterización de lavas vítreas de fuentes y sitios arqueológicos del Formativo Temprano en la subárea circumpuneña: resultados preliminares y proyecciones para la prehistoria atacameña. Estudios Atacameños 28:45-57.

Seelenfreund, A., E. Fonseca, F. Llona, L. Lera, C. Sinclaire y C. Rees. 2009. Geochemical analysis of vitreous rocks exploited during the Formative Period in the Atacama Region, Northern Chile. Archaeometry 51(1):1-25.

Shackley, M. S. 1998. Current issues and future directions in archaeological volcanic glass studies. En M. Steven Shackley (ed.), Archaeological obsidian studies: method and theory, Capítulo 1, pp. 1-14, Plenum Press, New York.

Somonte, C., L. Cohen. 2006. Reocupación y producción lítica: un aporte a la historia ocupacional de los recintos 3 y 4 del sitio agropastoril de Punta de la Peña 9-Sector III (Antofagasta de la Sierra, Catamarca, Argentina). Werken 9:135-158.

Sprovieri, M., M. D. Glascock. 2007. Aproximación a la circulación de obsidiana en el valle Calchaquí salteño entre los siglos IX y XV. Actas del XVI Congreso Nacional de Arqueología Argentina, Tomo III, pp. 221-226, S. S. de Jujuy.

Tarragó, M., L. González y J. Nastri. 1997. Las interacciones prehispánicas a través del estilo: el caso de la iconografía santamariana. Estudios Atacameños 14:223-242.

Yacobaccio, H. D., P. S. Escola, F. Pereyra, M. Lazzari y M. D. Glascock. 2004. Quest for ancient routes: obsidian sourcing research in Northwestern Argentina. Journal of Archaeological Science 31(2):193-204. 